\title{
Carbon-Involved Near-Surface Evolution of Cobalt Catalyst during Reaction: An in-situ Study
}

\section{Feng Yang}

Beijing National Laboratory for Molecular Science, Key Laboratory for the Physics and Chemistry of Nanodevices, College of Chemistry and Molecular Engineering, Peking University https://orcid.org/00000002-3637-814X

\section{Haofei Zhao}

Beijing Advanced Innovation Center for Materials Genome Engineering, Beijing Key Laboratory for Magneto-Photoelectrical Composite and Interface Science, School of Mathematics and Physics, University of Science and Technology Beijing

\section{Wu Wang}

Department of Physics, Southern University of Science and Technology

\section{Qidong Liu}

Beijing National Laboratory for Molecular Science, Key Laboratory for the Physics and Chemistry of Nanodevices, College of Chemistry and Molecular Engineering, Peking University

\section{Xu Liu}

Beijing National Laboratory for Molecular Science, Key Laboratory for the Physics and Chemistry of Nanodevices, College of Chemistry and Molecular Engineering, Peking University

\section{Yuecong Hu}

Beijing National Laboratory for Molecular Science, Key Laboratory for the Physics and Chemistry of Nanodevices, College of Chemistry and Molecular Engineering, Peking University

\section{Xinrui Zhang}

Beijing National Laboratory for Molecular Science, Key Laboratory for the Physics and Chemistry of Nanodevices, College of Chemistry and Molecular Engineering, Peking University

\section{Dongsheng He}

Core Research Facilities, Southern University of Science and Technology

\section{Yingying Xu}

Beijing Advanced Innovation Center for Materials Genome Engineering, Beijing Key Laboratory for Magneto-Photoelectrical Composite and Interface Science, School of Mathematics and Physics, University of Science and Technology Beijing

\section{Jiaqing He}

Department of Physics, Southern University of Science and Technology

\section{Rongming Wang ( $\nabla$ rmwang@ustb.edu.cn )}

Beijing Advanced Innovation Center for Materials Genome Engineering, Beijing Key Laboratory for Magneto-Photoelectrical Composite and Interface Science, School of Mathematics and Physics, 
University of Science and Technology Beijing https://orcid.org/0000-0003-4075-6956

\section{Yan Li ( $\square$ yanli@pku.edu.cn )}

Beijing National Laboratory for Molecular Science, Key Laboratory for the Physics and Chemistry of Nanodevices, College of Chemistry and Molecular Engineering, Peking University https://orcid.org/00000002-3828-8340

\section{Article}

Keywords: cobalt catalyst, in-situ, catalytic performance

Posted Date: July 7th, 2020

DOI: https://doi.org/10.21203/rs.3.rs-36341/v1

License: (c) (1) This work is licensed under a Creative Commons Attribution 4.0 International License. Read Full License 


\section{Abstract}

When carbon-containing species are involved in reactions catalyzed by transition metals at high temperature, the diffusion of carbon on/in catalysts dramatically influence the catalytic performance. Acquiring information on the carbon-diffusion-involved evolution of catalysts at atomic level is crucial for understanding the reaction mechanism yet also challenging. For the chemical vapor deposition process of single-walled carbon nanotubes (SWCNTs), we developed methodologies to record in-situ the nearsurface structural and chemical evolution of Co catalysts with carbon permeation using an aberrationcorrected environmental transmission electron microscope and the synchrotron X-ray absorption spectroscopy. The nucleation and growth of SWCNTs were linked with the partial carbonization of catalysts and the alternating dissolvement-precipitation of carbon in catalysts. The dynamics of carbon atoms in catalysts brings deeper insight into the growth mechanism of SWCNTs and also sheds light on inferring mechanisms of more reactions. The methodologies developed here will find broad applications in studying catalytic and other processes.

\section{Background}

Transition metals ( $\mathrm{Fe}, \mathrm{Co}, \mathrm{Ni}, \mathrm{Cu}$, etc.) are in widespread use as heterogeneous catalysts for many reactions of both scientific and industrial interest. For example, such catalysts play a leading role in petrochemical engineering processes, including Fisher-Tropsch synthesis ${ }^{1-3}$, selective hydrogenation of alkyne $\mathrm{e}^{4,5}$, reforming and pyrolysis of methane ${ }^{6}$. Carbon atoms in transitional-metal catalysts may act as either promotors or poisoners. At low carbon content, $\mathrm{C}$ atoms can induce the formation of metal carbide intermediates, which then serve as catalytically active sites ${ }^{5,7-11}$. However, at high carbon content, graphite or coke can emerge on top of the metal surface, and even surround and encapsulate metal nanoparticles, fully blocking the active sites ${ }^{1}$.

The transition metals are also generally applied as high efficient catalysts for the synthesis of carbon nanomaterials, including SWCNTs ${ }^{12-20}$ and graphene ${ }^{21,22}$. The structure and composition of catalysts are crucially important and determinative for achieving high selectivity in diameter and chirality for SWCNT growth $^{13,14,23,24}$. During the CVD process, carbon atoms may diffuse on surface or into the body of transitional-metal nanoparticles, resulting in a diverse and heterogeneous composition and structure of the catalysts ${ }^{23-29}$. Hence, there are a long-time controversy on the active catalytic species. The carbon enrichment in metal nanoparticles also modify their electronic property and wetting property ${ }^{27}$, which in turn leads to different growth modes of SWCNTs on catalysts ${ }^{30}$. In the controlled growth of graphene, the selection of metal substrate with suitable affinity to carbon is also a key issue. A precise tuning of carbon solubility in catalysts by combining different transition metals precisely controlled the production of mono- or bi-layer graphene $\mathrm{e}^{22,31-33}$. These reports highlight the importance of the interaction between metal and carbon, which needs further investigation. The key challenge is to figure out the behavior of carbon atoms in metal catalysts, especially to obtain atomic scale and dynamic information under reactive environment. 
Techniques based on aberration-corrected environmental transmission electron microscope (AC-ETEM) ${ }^{34-}$ ${ }^{40}$ have shown to be a powerful tool for the investigation of such process at atomic spatial and high temporal resolution. With the help of in-situ imaging, the nucleation process of carbon nanotubes on both liquid and solid catalysts were recorded $25,29,35,41-43$. Nonetheless, much less is known about structural and compositional information at atomic scale on carbon involved catalyst surface and how nanotube nucleation is aroused from carbon-metal interaction.

Herein, taking the catalytic synthesis of SWCNTs as a model process, we investigate the diffusion behavior of carbon atoms in near-surface of cobalt catalysts under carbon feeding conditions by using AC-ETEM. The insufficient carbon supply is a general problem in ETEM study due to the low pressure in ETEM chamber and the tiny heating area of the in-situ chip-heating system. Here we used cobalt acetate $\left(\mathrm{Co}(\mathrm{OAc})_{2}\right)$ as both the catalyst precursor and the solid carbon source. The in-situ decomposition of acetate groups upon heating brings about a localized carbon feeding high enough to facilitate the growth of SWCNTs. Through atomic-scale visualization and elemental electron energy loss spectrum (EELS) analysis, we found that the cobalt catalyst was activated through carbon dissolving in near-surface and composed of $\mathrm{Co}_{-} \mathrm{Co}_{3} \mathrm{C}$. The dynamic structure evolution of catalyst revealed that carbon dissolving and precipitation in cobalt facilitated the growth of SWCNTs. These findings were also proved by in-situ ambient-pressure synchrotron X-ray absorption spectroscopy (XAS).

\section{Results}

In-situ observation of the growth of SWCNTs from cobalt catalysts. The in-situ experiments of SWCNT growth were conducted on an aberration-corrected FEI Titan G2 80-300 ETEM microscope (operated at $80 \mathrm{kV}$ ) with a resolution of $1.0 \AA$. The ETEM chip with amorphous $\mathrm{SiO}_{2} / \mathrm{SiN}_{x}$ membrane was mounted onto a Micro Electron-Mechanical System based micro heater (ThermoFisher Scientific). The cobalt acetate dispersed on $\mathrm{SiO}_{2} / \mathrm{SiN}_{x}$ membrane of ETEM chip was annealed in vacuum $\left(\sim 10^{-5} \mathrm{~Pa}\right)$ at $600-$ $750^{\circ} \mathrm{C}$ for $10 \mathrm{~min}$. The cobalt acetate was decomposed into $\mathrm{CoO}$ and further transformed into Co as revealed by the in-situ selected-area electron diffraction patterns shown in Supplementary Fig. 1. In this process, the carbon species or other carbon-hydrogen species generated from the decomposition of acetate groups took the roles of reductants. Meanwhile, the carbon species decomposed from acetate groups could also act as carbon source ${ }^{44}$ to feed the growth of SWCNTs.

Figure 1a-d shows a sequenced frame extracted from a video of a single shell graphitic cap nucleated from a $\sim 4 \mathrm{~nm}$ Co nanoparticle. At a moment designated as $0 \mathrm{~s}$, graphitic shell formed and encapsulated the Co nanoparticle, with a pair of diffraction spots indicated by boxes in fast Fourier transformation (FFT) corresponding to $\{111\}$ lattice spacing of face-centered-cubic (fcc) Co (Fig. 1e). At $41 \mathrm{~s}$, the Co nanoparticle became structurally inhomogeneous. Two new pairs of diffraction spots indicated by circles, $2.5 \AA$ and $2.0 \AA$ in spacing with $90^{\circ}$ interplanar angle, appeared and well accorded with the diffraction pattern of $\mathrm{Co}_{3} \mathrm{C}$ carbide (ICSD 617389, space group: Pnma, $a=5.07 \AA, b=6.70 \AA ., c=4.53 \AA, a=\beta=\gamma=90^{\circ}$ ) (Fig. 1f, Supplementary Table 1). Indeed, they could not be assigned to either fcc/hexagonal-close- 
packing (hcp) Co or $\mathrm{Co}_{2} \mathrm{C}$. Then the graphite shell lifted off at $59 \mathrm{~s}$ to form a cap with a size of $\sim 1 \mathrm{~nm}$ (Fig. 1c, d, g, h). The active catalyst nanoparticle kept structurally inhomogeneous with co-existence of Co and $\mathrm{Co}_{3} \mathrm{C}$ phases. To avoid the effect of e-beam irradiation, we also performed the nanotube growth with e-beam off and then captured the ETEM images with e-beam on. Figure $1 \mathrm{i}$, j show typical single-shot ETEM images of individual SWCNTs nucleating from cobalt catalysts at $600^{\bullet} \mathrm{C}$. The FFT derived from the interface of catalyst-nanotube also showed the diffraction spots of metallic $\mathrm{Co}$ and $\mathrm{Co}_{3} \mathrm{C} \mathrm{(Fig.} 1 \mathrm{k}$ ). More active catalysts containing $\mathrm{Co}$ and $\mathrm{Co}_{3} \mathrm{C}$ were shown in Supplementary Fig. 2 and 3. Our observations in ETEM demonstrated generally that inhomogeneous $\mathrm{Co}^{-} \mathrm{Co}_{3} \mathrm{C}$ was active species for nucleating SWCNTs.

To gain deep insights into the growth dynamics of SWCNTs, we linked the catalyst evolution to the nucleation rate of SWCNTs (Fig. 1l, m). The nucleation rate was plotted as a function of the SWCNT length to growth time (Fig. $1 \mathrm{~m}$ ). The catalyst structure of each snap point was determined from FFT of time-sequenced ETEM images (Supplementary Fig. 2). It is obvious that the rate-limiting step was the incubation of graphitic shell on metallic Co nanoparticle, which lasted near $120 \mathrm{~s}$. The catalyst maintained the metallic state without $\mathrm{Co}_{3} \mathrm{C}$ species during the incubation process. Then in the follow-up stages, the formation of structurally inhomogeneous $\mathrm{Co}^{-} \mathrm{Co}_{3} \mathrm{C}$ at $\sim 120 \mathrm{~s}$ resulted in rapid elongation of carbon cap (Fig. $1 \mathrm{~m}$ ). After that, the active catalyst nanoparticle kept inhomogeneous containing Co and $\mathrm{Co}_{3} \mathrm{C}$. These observations indicate that the presence of $\mathrm{Co}_{3} \mathrm{C}$ might be essential in promoting the nucleation of cap and its elongation.

To further evaluate the general role of catalysts, we performed the X-ray photoelectron spectroscopy (XPS) to probe the composition of catalysts after growth of SWCNTs (Supplementary Fig. 4). The spectra were recorded at around Co $2 p$ core levels, where the background component was subtracted by the Shirley method. The two peaks with low intensity appearing at 778.3 and $793.7 \mathrm{eV}$ could be ascribed to Co $2 p_{3 / 2}$ and Co $2 p_{1 / 2}$ of metallic Co. Co $2 p_{3 / 2}$ peak at $781.3 \mathrm{eV}$ with satellite peak at $786.2 \mathrm{eV}$ were attributed to $\mathrm{Co}_{3} \mathrm{C}$ (Fig. 2a). Spin-orbital splitting between Co $2 \mathrm{p}_{3 / 2}$ and $2 \mathrm{p}_{1 / 2}$ is $15.8 \mathrm{eV}$, which further indicates the existence of $\mathrm{Co}$ (II) species ${ }^{45}$. It is obvious that $\mathrm{Co}_{3} \mathrm{C}$ and $\mathrm{Co}$ co-exist in catalysts. XAS was also used to investigate the local coordination structure of cobalt catalysts. In-situ X-ray absorption nearedge spectroscopy of $\mathrm{MgO}$ supported Co catalysts recorded at Co K-edge was collected under the atmospheric pressure $\mathrm{H}_{2}$ and $\mathrm{CH}_{4}$ at $700{ }^{\circ} \mathrm{C}$, respectively. The extended $\mathrm{X}$-ray absorption fine structure (EXAFS) oscillation of Co under $\mathrm{H}_{2}$ showed a first nearest-coordination peak of $2.1 \AA \AA$ observed from $R$ space, indicating the reduction of cobalt oxide. The first three nearest-coordination peaks of 1.5, 2.1, and 2.6 $\AA$ appeared when switching to $\mathrm{CH}_{4}$ atmosphere, which could be attributed to $\mathrm{Co}-\mathrm{C}$, Co-Co, and $\mathrm{Co}-\mathrm{C}$ based on fitting result, respectively (Fig. 2b). All above in/ex-situ X-ray spectroscopic results were consistent with ETEM observations that the Co catalysts were partially carbonized to form $\mathrm{Co}^{-} \mathrm{Co}_{3} \mathrm{C}$ during the growth of SWCNTs. 
Local carbonization of Co by carbon spatial permeation. To figure out the distribution of $\mathrm{Co}_{3} \mathrm{C}$ species in active catalyst nanoparticles during the growth of SWCNTs, we carried out further investigation on the interface of catalyst-nanotube. Figure $3 a$ shows a single shot AC-ETEM image of a nanotube growing from a cobalt nanocrystal at $600^{\circ} \mathrm{C}$. FFT of this seed nanoparticle was identified as inhomogeneous Co$\mathrm{Co}_{3} \mathrm{C}$ (Fig. 3b), which coincides with previous observations. By applying masks to the series of spots of Co marked with circles, the resulting inversed FFT (IFFT) showed the region of fcc-Co, which covers the entire catalyst (Fig. $3 \mathrm{c}$ ). However, IFFT images derived from the series of spots of $\mathrm{Co}_{3} \mathrm{C}$ marked with boxes showed the uneven distribution of $\mathrm{Co}_{3} \mathrm{C}$ in catalyst (Fig. 3d). Additionally, we found from IFFT images that $\mathrm{Co}_{3} \mathrm{C}$ region was close to the nucleation site of nanotube (Fig. 3d, marked by circles). More in-situ data were shown in Supplementary Fig. 3 , which all delivered similar results that $\mathrm{Co}_{3} \mathrm{C}$ species tended to aggregate around the nucleation site of nanotubes.

To quantitatively evaluate the carbon dissolved in catalysts, we made a statistical analysis of the lattice spacings of the outer layers of catalysts. Figure 4a shows a high-resolution AC-ETEM image of a graphitic cap on Co catalyst at $600^{\circ} \mathrm{C}$. The corresponding FFT marks a resolution information of $1.1 \AA$. We assigned atomic columns to hcp-Co [0001] and $\mathrm{CO}_{3} \mathrm{C}$ phases accordingly (Fig. 4b), which confirms again the co-existence of $\mathrm{Co}$ and $\mathrm{Co}_{3} \mathrm{C}$. A fit of two dimensional (2D) Gaussians to the intensity of distribution of the atomic columns was used to acquire the precise column coordinates, which improved the resolution to sub-angstrom ${ }^{38,46}$. The perpendicular layer spacings were determined locally from those fitted coordinates and then averaged for each shell $\left(d_{12}, d_{23}, d_{34} \ldots\right)$ (Supplementary Fig. 5). Figure 4c

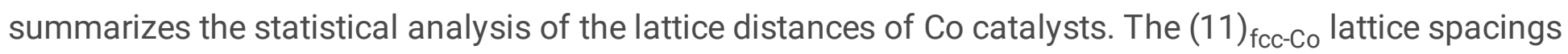
of the active catalyst nanoparticle with a cap gradually increased from $2.15( \pm 0.06) \AA$ to $2.58( \pm 0.17) \AA$ from core region to the outermost surface. Such a $\sim 37 \%$ lattice expansion of the surface layer could be induced by the carbon atoms dissolving into Co nanoparticle. We also analyzed the near surface lattice spacings of other active catalysts of either fcc or hcp-Co phase. A general lattice expansion of 14 37\% from the outmost surface toward the core was observed in all the active catalysts (Fig. 4d). For comparison, we also measured the near-surface layer spacings of inactive catalyst without CNT nucleation. It was found that only the outermost surface $\left(d_{12}=2.17 \pm 0.05 \AA\right)$ displayed a larger spacing and the inner lattice spacings $\left(d_{23}, d_{34}, d_{45} \ldots\right)$ were close to theoretical value $\left(d_{(11) C_{0}}=2.06 \AA\right)$ (Fig. $4 \mathrm{c}$, Supplementary Fig. 6). This finding indicates that carbon atoms did not diffuse into inner part of inactive Co nanoparticles.

We also compared the lattice expansion from the different directions of active catalysts with SWCNTS (Fig. 4e-h). The near-surface crystal lattice was significantly expanded near the nucleation site of SWCNT (Fig. 4e, f). This finding could originate from the diffusion of carbon atoms near the region where the SWCNT grows, which was consistent with above IFFT results (Fig. 3d). However, the spacings away from the nucleation site were not expanded, which is similar to the inactive catalysts (Fig. $4 \mathrm{~g}, \mathrm{~h}$ ).

To direct visualize the carbon dissovled in catalysts, in-situ scanning TEM (STEM)-EELS was collected on an acitve catalyst with a CNT grown at $600{ }^{\circ} \mathrm{C}$ (Fig. 5a, b). The nanoparticle slightly rotated after EELS 
mapping due to the e-beam irradiation. STEM-EELS elemental mapping showed that carbon element distributed in both CNT and the near-surface of catalyst nanoparticle, whereas cobalt element homogeneously distributed in the whole catalyst (Fig. $5 \mathrm{c}-\mathrm{e}$ ). The line scan profile of EELS across the CNT-catalyst interface clearly showed co-existence of $C$ and $C o$ in the near-surface of catalyst (Fig. $5 f, g$, layer 3-6). The intensity of energy loss peaks of $C K$-edge decreased from catalyst surface to body, whereas the Co $L_{2,3}$-edge showed the inverse tendency (Fig. 5 g, layer 3-12 and Fig. $5 \mathrm{~h}$ ). For comparison, we also collected the in-situ EELS on inactive catalyst without CNT nucleation, showing the absence of carbon signal in Co catalyst, i.e., no carbon diffusion into catalyst (Supplementary Fig. 7).

All the above in-situ atomic scale and elemental characterizations as well as quantitative analysis supported that carbon atoms heavily dissolved in near-surface (thickness: 1 3 nm) of active Co nanoparticles and consequently forming $\mathrm{Co}_{3} \mathrm{C}$ during the growth of SWCNTs.

\section{Dynamics of carbon dissolving and precipitation in catalyst during the nucleation of carbon cap. Figure} $6 a-c$ showed the time-sequenced ETEM images of carbon cap growing on a cobalt nanoparticle at 700 - $\mathrm{C}$. At the relative time defined as $0 \mathrm{~s}$, the carbon cap started to nucleate on surface of Co nanoparticle with a height of $2.6 \AA$. Then the cap gradually grew larger and lifted off from the catalyst surface at 975 $\mathrm{s}$. All the corresponding FFT derived from the near-surface of the catalyst evidenced the co-existence of $\mathrm{Co}$ and $\mathrm{Co}_{3} \mathrm{C}$ (Fig. $6 \mathrm{~d}-\mathrm{e}$ ). The IFFT images derived from the diffraction spots of $\mathrm{Co}_{3} \mathrm{C}$ showed that the $\mathrm{Co}_{3} \mathrm{C}$ species rotated during growth of carbon cap. The orientation of $\mathrm{Co}_{3} \mathrm{C}$ changed from [21] to [12] by $41.5^{\bullet}$, and further changed to [10] by $33.8^{\bullet}$ (Fig. $6 \mathrm{~g}-\mathrm{i}$ ). However, the orientation of metallic Co species maintained stable (Fig. $6 \mathrm{j}-\mathrm{I}$ ). Figure $6 \mathrm{~m}$ summarized the $(11)_{\text {Co }}$ spacings at different exposure time. At the time of $0 \mathrm{~s}$, we observed a $\sim 37 \%$ lattice expansion from outermost surface to core region of Co nanoparticle. This data has been shown in Fig. 4c. However, this tendency reversed at $975 \mathrm{~s}$ that the lattice spacings decreased from core region to outermost surface (Fig. $6 \mathrm{~m})$. Such a structural fluctuation induced by the lattice expansion and shrink in near-surface could be related to carbon atoms' dissolving and precipitation in Co nanoparticle. The precipitation of carbon atoms was responsible for the nucleation and growth of the carbon cap.

\section{Discussion}

To further understand the role of Co catalysts in nucleating the SWCNTs, we also analyzed the poisoned catalysts, which are inactive for the growth of nanotubes. We observed that these poisoned catalyst nanoparticles were either entirely encapsulated by the graphitic shell or coated by amorphous carbon. For those nanoparticles entirely encapsulated by the graphitic shells, they still maintained the composition of metallic $\mathrm{Co}$ and did not form cobalt carbides $\left(\mathrm{Co}_{2} \mathrm{C}\right.$ or $\left.\mathrm{Co}_{3} \mathrm{C}\right)$ (Supplementary Fig. 8a-f). This could be attributed to that the metallic Co nanoparticles were wrapped by the graphitic shells, thus carbon atoms could not further diffuse into $\mathrm{Co}$ to form $\mathrm{Co}_{3} \mathrm{C}$. The pure $\mathrm{Co}_{3} \mathrm{C}$ carbide nanoparticles were coated by amorphous carbon instead of graphitic shells (Supplementary Fig. $8 \mathrm{~g}-\mathrm{j}$ ). This indicated that pure $\mathrm{Co}_{3} \mathrm{C}$ was favorable for the precipitation of amorphous carbon but not effective in catalyzing the formation of 
graphitic $s p^{2}$ carbon. These observations about inactive catalysts further verified that the growth of SWCNTs was realized only by the cooperation of $\mathrm{Co}$ and $\mathrm{Co}_{3} \mathrm{C}$.

To summarize, aberration-corrected ETEM has been used in combination with quantitative 2D Gaussian fitting, in-situ EELS, and XAS analysis, respectively, to systematically investigate at atomic scale the behavior of Co nanoparticles under carbon feeding and the nucleation and growth of SWCNTs. The inhomogeneous $\mathrm{Co}_{-} \mathrm{CO}_{3} \mathrm{C}$ was evidenced as catalytically active species for the growth of SWCNTs. The $\mathrm{Co}_{3} \mathrm{C}$ species were structurally fluctuated in near-surface of Co catalysts induced by carbon diffusion and precipitation, acting as carbon carriers in the growth of SWCNTs. These findings elucidate the dynamic behavior of carbon in transitional-metal nanoparticles at the atomic scale and reveal the general importance of carbon permeation in metal in hydrocarbon or other carbon-containing species involved heterogeneous reactions. Our in-situ methodology based on atomic-scale ETEM imaging and elemental spectroscopy is shown to be a powerful tool for the investigation of light elements in solids and their dynamics in real time and direct space. Such in-situ methodologies should be valid for the mechanistic investigation of a wide variety of metal catalyzed reactions and other processes such as crystallization and alloying.

\section{Methods}

ETEM experiments. The in-situ study was conducted by using an aberration-corrected Titan G2 80-300 ETEM equipped with a MEMS based micro heater. The catalyst precursor of cobalt acetate $\left(\mathrm{Co}(\mathrm{OAC})_{2}\right)$ dissolved in ethanol $\left(0.1 \mathrm{mmol} \bullet \mathrm{L}^{-1}\right)$ was dropped onto the ETEM chips. We used two methods to prepare Co catalyst and perform in-situ SWCNT growth. (i) The as-prepared specimen was first heated to $600{ }^{\circ} \mathrm{C}$ for $30 \mathrm{~s}$ and stabilized in high vacuum $\left(\sim 10^{-5} \mathrm{~Pa}\right)$ at $600{ }^{\circ} \mathrm{C}$ for $10 \mathrm{~min}$ with e-beam off. After that, TEM images and videos were captured with e-beam on. During annealing at $600^{\circ} \mathrm{C}$, the cobalt acetate decomposed to metallic Co nanoparticles, carbon, and other reductive species $\left(\mathrm{CO}, \mathrm{C}_{2} \mathrm{H}_{6}, \mathrm{H}_{2}\right)$. The carbon containing species could act as carbon source to feed the growth of carbon nanotubes. (ii) The asprepared specimen was first heated to $500{ }^{\circ} \mathrm{C}$ in pure $\mathrm{O}_{2}(50 \mathrm{~Pa})$ for $30 \mathrm{~min}$ to form $\mathrm{Co}_{3} \mathrm{O}_{4}$ and reduced in $\mathrm{H}_{2}(50 \mathrm{~Pa})$ at $700{ }^{\circ} \mathrm{C}$ for 30 min to form metallic Co catalysts. Then $\mathrm{CH}_{4}(99.99 \%)$, or $\mathrm{CH}_{4} / \mathrm{H}_{2}$ $(\mathrm{v} / \mathrm{v}=7 / 3)$, or CO $(99.999 \%)$ was introduced to TEM chamber to grow SWCNTs at $600-750{ }^{\circ} \mathrm{C}$ in a pressure of $28-503 \mathrm{~Pa}$.

STEM-EELS experiments. In-situ STEM EELS data were collected in another, but otherwise the same aberration-corrected Titan 80-300 ETEM. It was equipped a Gatan image filter (Quantum 936) with an energy dispersion of $0.25 \mathrm{eV}$ operated at an acceleration voltage of $300 \mathrm{kV}$. The zero-loss EELS was acquired immediately after obtaining the core-loss EELS on individual nanoparticle. The obtained EELS data were further analyzed in Digital Micrograph software. The position of core-loss EELS was corrected with the corresponding zero-loss peak and the extrapolated background was then subtracted from the 
edge of interest. A Fourier-ratio deconvolution was further performed to remove the effect of plural scattering.

2D Gaussian fitting of TEM image. The 2D Gaussian fit to the intensity distribution of the atomic columns from HRTEM image was conducted using the StatSTEM V3.0 code run on MATLAB (R2015b) ${ }^{46}$. More details were showed in Supplementary Fig. 5 and corresponding discussion.

EX-situ XPS. The XPS experiments were performed using an AXIS Supra/Ultra Imaging X-ray Photoelectron Spectrometer. The binding energy of the XPS spectra was calibrated to that of C 1s (284.6 $\mathrm{eV})$. It was performed and exposed a monochromated Al Ka $(1486.6 \mathrm{eV})$. We prepared Co catalysts and perform low-pressure $(1.79 \mathrm{kPa})$ ethanol-CVD growth of SWCNTs at $900^{\circ} \mathrm{C}$. SEM image of as-grown SWCNTs can be seen in Supplementary Fig. 4.

In-situ synchrotron X-ray absorption spectroscopy. The XAFS spectra at Co K-edge were obtained at BL14W-1 beamline of Shanghai Synchrotron Radiation Facility operated at $3.5 \mathrm{GeV}$. For in-situ XANES measurements, a homemade CVD apparatus was equipped in BL14W-1. The quartz tube was strictly parallel to beam direction. The MgO supported Co catalyst (Co $5 \mathrm{wt} \%$ ) thin flake was inserted in quartz tube, facing the beam direction at $90^{\bullet}$. The in-situ Co K-edge XANES spectra were collected in a transmission mode at $700^{\circ} \mathrm{C}$ with the energy range of $7513-8509 \mathrm{eV}$, under the atmospheric pressure of $\mathrm{H}_{2} / \mathrm{He}\left(10 \% \mathrm{H}_{2}\right)\left(200 \mathrm{~cm}^{3} \cdot \mathrm{min}^{-1}\right)$ and $\mathrm{CH}_{4}\left(20 \mathrm{~cm}^{3} \cdot \mathrm{min}^{-1}\right)$, respectively. The data were interrupted using Athena and Artemis codes to remove background, normalize edge-step, and fit the EXAFS data using standard procedures. The absorption edge of Co foil $\left(E_{0}=7709 \mathrm{eV}\right)$ was used to calibrate the energy edge of all samples.

Data availability. The data that support the plots within this paper and other findings of this study are available from the corresponding author upon reasonable request.

\section{References}

1 de Smit, E. \& Weckhuysen, B. M. The renaissance of iron-based Fischer-Tropsch synthesis: on the multifaceted catalyst deactivation behaviour. Chem. Soc. Rev. 37, 2758-2781 (2008).

2 Rahmati, M., Safdari, M.-S., Fletcher, T. H., Argyle, M. D. \& Bartholomew, C. H. Chemical and Thermal Sintering of Supported Metals with Emphasis on Cobalt Catalysts During Fischer-Tropsch Synthesis. Chem. Rev. 120, 4455-4533 (2020). 
3 Yang, C., Zhao, H. B., Hou, Y. L. \& Ma, D. $\mathrm{Fe}_{5} \mathrm{C}_{2}$ Nanoparticles: A Facile Bromide-Induced Synthesis and as an Active Phase for Fischer-Tropsch Synthesis. J. Am. Chem. Soc. 134, 15814-15821 (2012).

4 Studt, F. et al. Identification of Non-Precious Metal Alloy Catalysts for Selective Hydrogenation of Acetylene. Science 320, 1320-1322 (2008).

5 Teschner, D. et al. The roles of subsurface carbon and hydrogen in palladium-catalyzed alkyne hydrogenation. Science 320, 86-89 (2008).

6 Upham, D. C. et al. Catalytic molten metals for the direct conversion of methane to hydrogen and separable carbon. Science 358, 917-920 (2017).

7 de Smit, E. et al. Stability and Reactivity of epsilon-chi-theta Iron Carbide Catalyst Phases in FischerTropsch Synthesis: Controlling mu(c). J. Am. Chem. Soc. 132, 14928-14941 (2010).

8 Zhong, L. et al. Cobalt carbide nanoprisms for direct production of lower olefins from syngas. Nature 538, 84-87 (2016).

9 Liu, X. et al. Environmental Transmission Electron Microscopy (ETEM) Studies of Single Iron Nanoparticle Carburization in Synthesis Gas. ACS Catal. 7, 4867-4875 (2017).

10 Pique, O. et al. Subsurface Carbon: A General Feature of Noble Metals. Angew. Chem. Int. Ed. 58, 1744-1748 (2019).

11 Rinaldi, A. et al. Dissolved carbon controls the initial stages of nanocarbon growth. Angew. Chem. Int. Ed. 50, 3313-3317 (2011).

12 Harutyunyan, A. R. et al. Preferential Growth of Single-Walled Carbon Nanotubes with Metallic Conductivity. Science 326, 116-120 (2009).

13 Chiang, W. H. \& Sankaran, R. M. Linking Catalyst Composition to Chirality Distributions of as-Grown Single-Walled Carbon Nanotubes by Tuning $\mathrm{Ni}_{\mathrm{x}} \mathrm{Fe}_{1-\mathrm{x}}$ Nanoparticles. Nat. Mater. 8, 882-886 (2009).

14 Yang, F. et al. Chirality-Specific Growth of Single-Walled Carbon Nanotubes on Solid Alloy Catalysts. Nature 510, 522-524 (2014).

15 Sanchez-Valencia, J. R. et al. Controlled synthesis of single-chirality carbon nanotubes. Nature 512, 61-64 (2014).

16 Zhao, Q., Xu, Z., Hu, Y., Ding, F. \& Zhang, J. Chemical vapor deposition synthesis of near-zigzag singlewalled carbon nanotubes with stable tube-catalyst interface. Sci. Adv. 2, 1501729 (2016).

17 Yang, F. et al. Chirality Pure Carbon Nanotubes: Growth, Sorting, and Characterization. Chem. Rev. 120, 2693-2758 (2020). 
18 Wang, H. et al. Catalysts for Chirality Selective Synthesis of Single-Walled Carbon Nanotubes. Carbon 81, 1-19 (2015).

19 Xiang, R. \& Maruyama, S. Revisiting behaviour of monometallic catalysts in chemical vapour deposition synthesis of single-walled carbon nanotubes. Roy. Soc. Open Sci. 5, 180345 (2018).

20 Magnin, Y., Amara, H., Ducastelle, F., Loiseau, A. \& Bichara, C. Entropy-driven stability of chiral singlewalled carbon nanotubes. Science 362, 212-215 (2018).

21 Ago, H. et al. Epitaxial Chemical Vapor Deposition Growth of Single-Layer Graphene over Cobalt Film Crystallized on Sapphire. ACS Nano 4, 7407-7414 (2010).

22 Dai, B. et al. Rational design of a binary metal alloy for chemical vapour deposition growth of uniform single-layer graphene. Nat. Commun. 2, 522 (2011).

$23 \mathrm{An}, \mathrm{H}$. et al. Atomic-scale structural identification and evolution of Co-W-C ternary SWCNT catalytic nanoparticles: High-resolution STEM imaging on $\mathrm{SiO}_{2}$. Sci. Adv. 5, 9459 (2019).

24 Zhang, S. et al. Arrays of horizontal carbon nanotubes of controlled chirality grown using designed catalysts. Nature 543, 234-238 (2017).

25 Zhang, L. et al. Growth Termination and Multiple Nucleation of Single-Wall Carbon Nanotubes Evidenced by In Situ Transmission Electron Microscopy. ACS Nano 11, 4483-4493 (2017).

26 Lin, P. A. et al. Direct evidence of atomic-scale structural fluctuations in catalyst nanoparticles. J. Catal. 349, 149-155 (2017).

27 Diarra, M., Zappelli, A., Amara, H., Ducastelle, F. \& Bichara, C. Importance of Carbon Solubility and Wetting Properties of Nickel Nanoparticles for Single Wall Nanotube Growth. Phys. Rev. Lett. 109, 185501 (2012).

28 Ding, F. et al. The importance of strong carbon-metal adhesion for catalytic nucleation of singlewalled carbon nanotubes. Nano Lett. 8, 463-468 (2008).

29 Cao, K. et al. Comparison of atomic scale dynamics for the middle and late transition metal nanocatalysts. Nat. Commun. 9, 3382 (2018).

30 Fiawoo, M. F. et al. Evidence of Correlation between Catalyst Particles and the Single-Wall Carbon Nanotube Diameter: A First Step towards Chirality Control. Phys. Rev. Lett. 108, 195503 (2012).

31 Huang, M. \& Ruoff, R. S. Growth of Single-Layer and Multilayer Graphene on Cu/Ni Alloy Substrates. Acc. Chem. Res. 53, 800-811 (2020). 
32 Huang, M. et al. Large-area single-crystal AB-bilayer and ABA-trilayer graphene grown on a $\mathrm{Cu} / \mathrm{Ni}(111)$ foil. Nat. Nanotechnol. 15, 289-295 (2020).

$33 \mathrm{Wu}, \mathrm{T}$. R. et al. Fast growth of inch-sized single-crystalline graphene from a controlled single nucleus on Cu-Ni alloys. Nat. Mater. 15, 43-47 (2016).

34 Su, D. S., Zhang, B. \& Schlögl, R. Electron Microscopy of Solid Catalysts-Transforming from a Challenge to a Toolbox. Chem. Rev. 115, 2818-2882 (2015).

35 Helveg, S. et al. Atomic-scale imaging of carbon nanofibre growth. Nature 427, 426-429 (2004).

36 Yoshida, H. et al. Visualizing Gas Molecules Interacting with Supported Nanoparticulate Catalysts at Reaction Conditions. Science 335, 317-319 (2012).

37 Luo, L. et al. Atomic origins of water-vapour-promoted alloy oxidation. Nat. Mater. 17, 514-518 (2018).

38 Yang, F. et al. Atomic Scale Stability of Tungsten-Cobalt Intermetallic Nanocrystals in Reactive Environment at High Temperature. J. Am. Chem. Soc. 141, 5871-5879 (2019).

39 Zhang, X. et al. Revealing the Hidden Face of Ni@Au Core-Shell Catalyst During Reaction. Nat. Catal. 3, 411-417 (2020).

40 Wang, R. The dynamic of the peel. Nat. Catal. 3, 333-334 (2020).

41 Picher, M., Lin, P. A., Gomez-Ballesteros, J. L., Balbuena, P. B. \& Sharma, R. Nucleation of Graphene and Its Conversion to Single-Walled Carbon Nanotubes. Nano Lett. 14, 6104-6108 (2014).

42 Rodríguez-Manzo, J. A. et al. In situ nucleation of carbon nanotubes by the injection of carbon atoms into metal particles. Nat. Nanotechnol. 2, 307-311 (2007).

$43 \mathrm{He}, \mathrm{M}$. et al. Chiral-Selective Growth of Single-Walled Carbon Nanotubes on Lattice-Mismatched Epitaxial Cobalt Nanoparticles. Sci. Rep. 3, 1460 (2013).

44 Jia, K. et al. Copper-Containing Carbon Feedstock for Growing Superclean Graphene. J. Am. Chem. Soc. 141, 7670-7674 (2019).

45 Chen, Z., Liu, L. \& Chen, Q. One-pot template-free synthesis of urchin-like $\mathrm{Co}_{2} \mathrm{C} / \mathrm{Co}_{3} \mathrm{C}$ hybrid nanoparticles. Mater. Lett. 164, 554-557 (2016).

46 Pohl, D., Wiesenhutter, U., Mohn, E., Schultz, L. \& Rellinghaus, B. Near-surface strain in icosahedra of binary metallic alloys: segregational versus intrinsic effects. Nano Lett. 14, 1776-1784 (2014).

\section{Declarations}




\section{Acknowledgements}

We gratefully acknowledge Shanghai Synchrotron Radiation Facility of China (SSRF, Beamline 14W1), Prof. Ding Ma (Peking University) and Jingyuan Ma (SSRF) for the XAFS measurements, Core Research Facilities of Southern University of Science and Technology for ETEM-EELS measurements. We thank Dr. Kecheng Cao and Dr. Xiao Wang for the valuable comments on the manuscript. This work is financially supported by National Key Research and Development Program of China (2016YFA0201904 and 2018YFA0703700), National Natural Science Foundation of China (21631002 and 51971025), Shenzhen Basic Research Project (JCYJ20170817113121505), as well as Fundamental Research Funds for the Central Universities (FRF-BD-18-004A). J.H. acknowledges the Science and Technology Innovation Committee Foundation of Shenzhen (KQTD2016022619565991 and ZDSYS20141118160434515). F.Y. was supported in part by BMS Junior Fellow of Beijing National Laboratory for Molecular Science and Innovation Project for Guangdong Provincial Department of Education (2019KTSCX155).

\section{Author contributions}

F.Y., H.Z., R.W., and Y.L. conceived and designed the experiments. F.Y., H.Z., and W.W. performed the ETEM and EELS. F.Y., Q.L., and X.L. performed the XPS and XAFS. F.Y. and Y.L. wrote the manuscript. All authors analyzed the data, discussed the results, and approved the manuscript. F.Y. and H.Z. contributed equally to this work.

\section{Competing interests}

The authors declare no competing interests.

\section{Additional information}

Supplementary information is available for this paper at https://doi.org/10.1038/xxxxxx.

Reprints and permissions information is available at www.nature.com/reprints

Correspondence and requests for materials should be addressed to Y.L. or R.W.

\section{Figures}



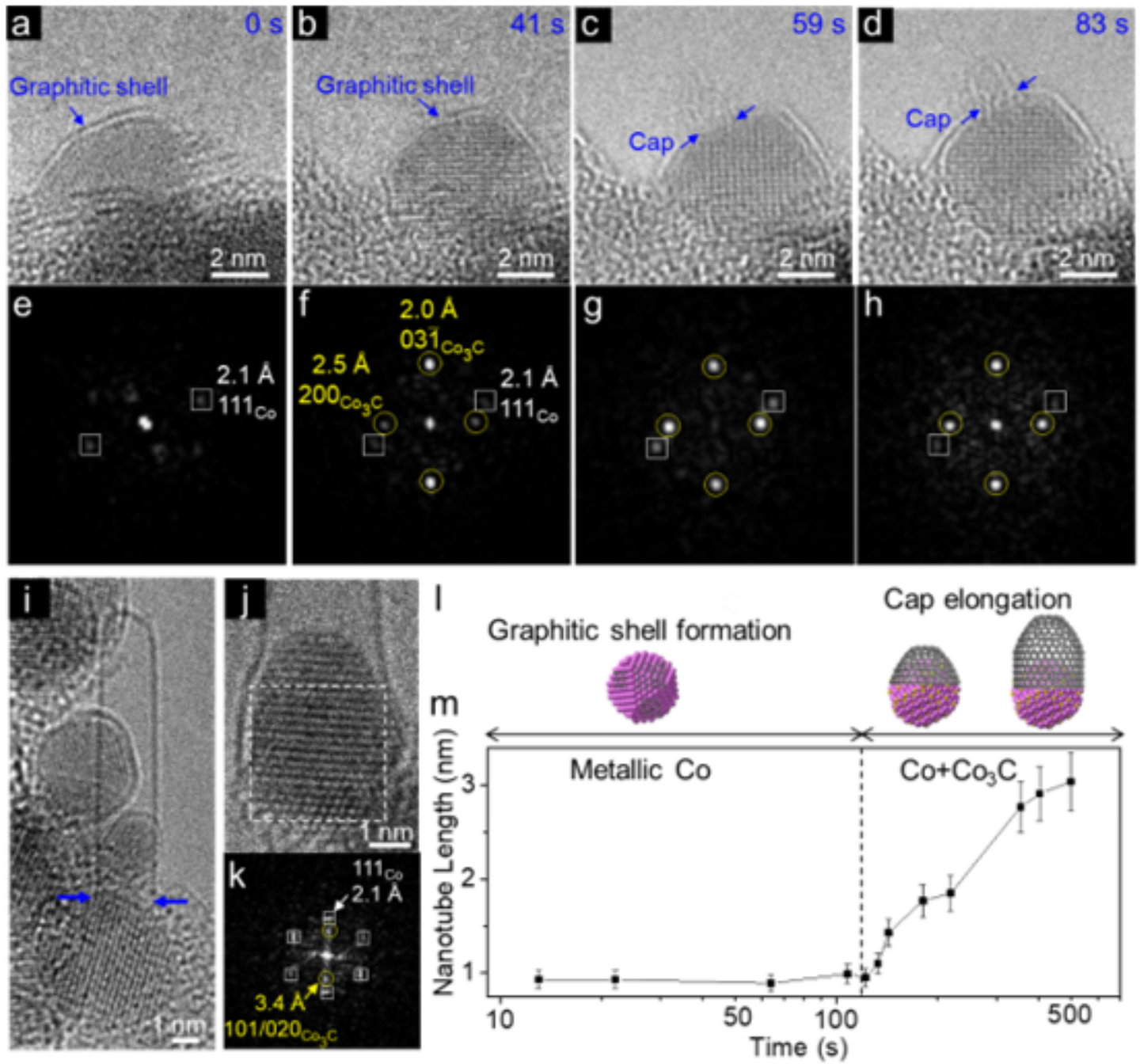

\section{Figure 1}

Nucleating SWCNTs from cobalt catalysts. a-h Time-sequenced AC-ETEM images of a cap growing from cobalt catalyst acquired at 750 C $(a-d)$ and corresponding FFT of the particle region $(e-h)$. i-k ACETEM images of SWCNTs grown on catalysts $(i, j)$ and FFT $(k)$ of the particle region marked in $(j)$. I Schemes showing each stage of nucleation and corresponding catalyst species. $m$ Length of the cap as a function of growth time. Catalyst composition was determined from the FFT in each snap point.
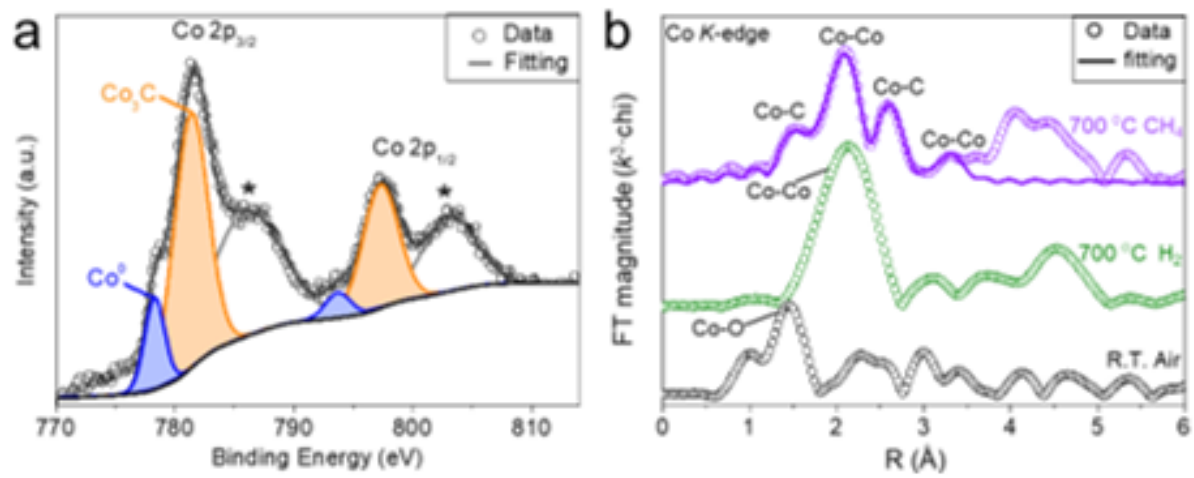

Figure 2 
X-ray photoelectron and absorption spectroscopic characterizations of catalysts. a Ex-situ Co $2 p$ XPS of catalysts after the growth of SWCNTs on $\mathrm{Si} / \mathrm{SiO} 2$ substrate. The $\mathrm{CoO}$ and $\mathrm{Co} 3 \mathrm{C}$ chemical states were identified. The asterisk depicts the shake-up peak of the Co3C peak. b In-situ Co K-edge Fourier transforms of EXAFS of Co catalysts under $\mathrm{H} 2$ and $\mathrm{CH} 4$ at 700 C. C. $\mathrm{Co} 3 \mathrm{O} 4$ nanoparticles was used as referenced sample and performed at room temperature.
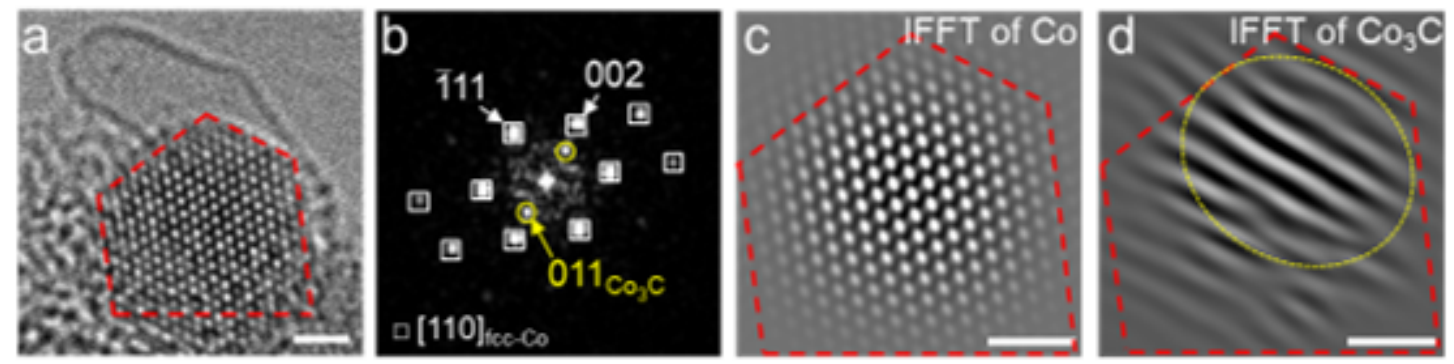

\section{Figure 3}

Distribution of Co3C species in active catalysts. a Single shot high-resolution ETEM image showing a SWCNT nucleating on cobalt catalyst acquired at 600 OC. b FFT of the particle region marked by dashes in (a). c, d IFFT formed using spots of $\mathrm{Co}$ (c) and Co3C (d) in corresponding FFT. Region of Co3C crystalline was roughly marked by circle in (d).
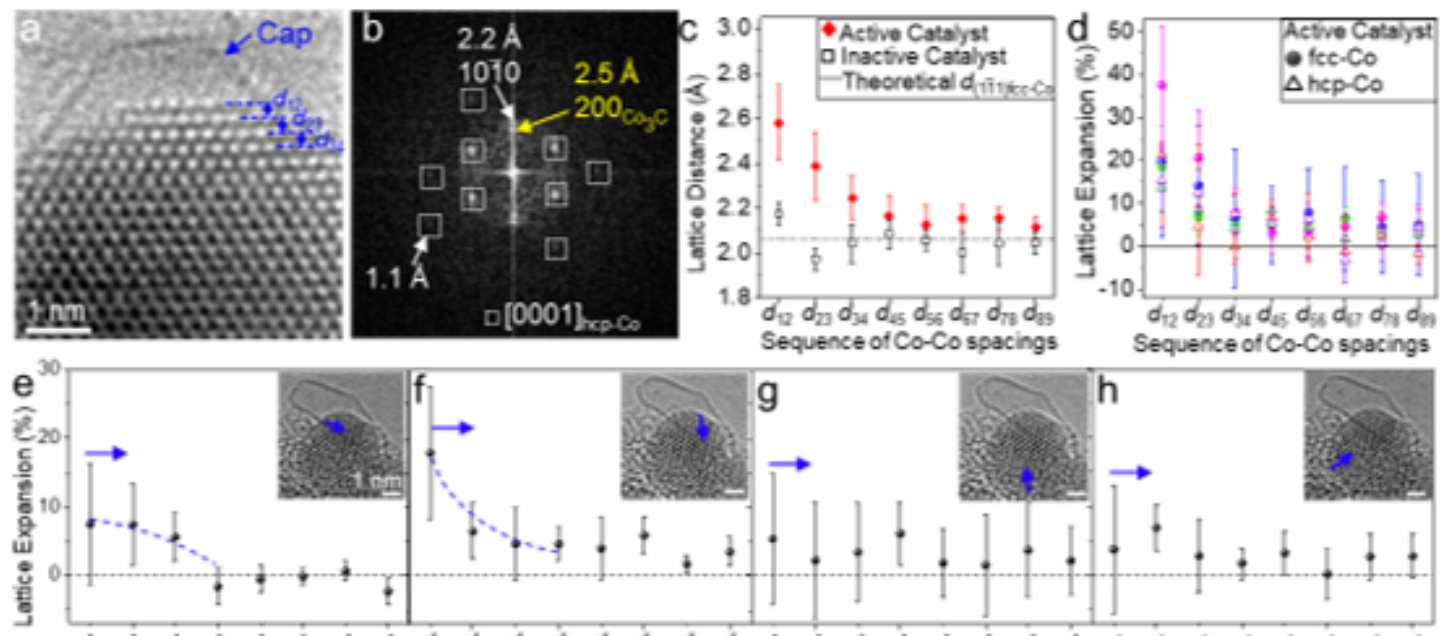

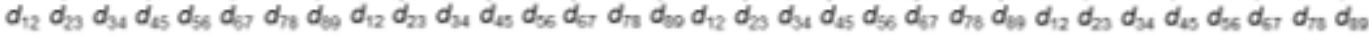
Sequence of $\mathrm{Co} \cdot \mathrm{Co}$ spacings

\section{Figure 4}

Quantatively analysis of carbon atom dissovling in active catalysts. a The representative Wiener filtered AC-ETEM image and determination of the position of individual atomic columns by 2D Gaussian fitting. The sequence of layer spacings is labeled as d12, d23, d34 ..., from surface to core. b Corresponding FFT of near-surface of this nanoparticle. c The representative (1 1) fcc-Co lattice spacings of active and inactive catalysts as a function of sequencial atomic numbers from the outermost surface to core region (d12, d23, d34...d89). d Summary of lattice expansion measured from both fcc and hcp Co active catalysts. The degree of lattice expansion was calculated by (dmeasured- 
dtheoretical)/dtheoretical $\times 100 \%$. e-h Near-surface lattice expansion measured from different directions of the same catalyst as shown in Fig. 3a. The arrows indicated the measured directions from outmost surface to core region (from d12 to d89). All error bars were calculated from the standard deviation of the mean lattice parameters among 6-12 individual measurements for a given atomic shell.

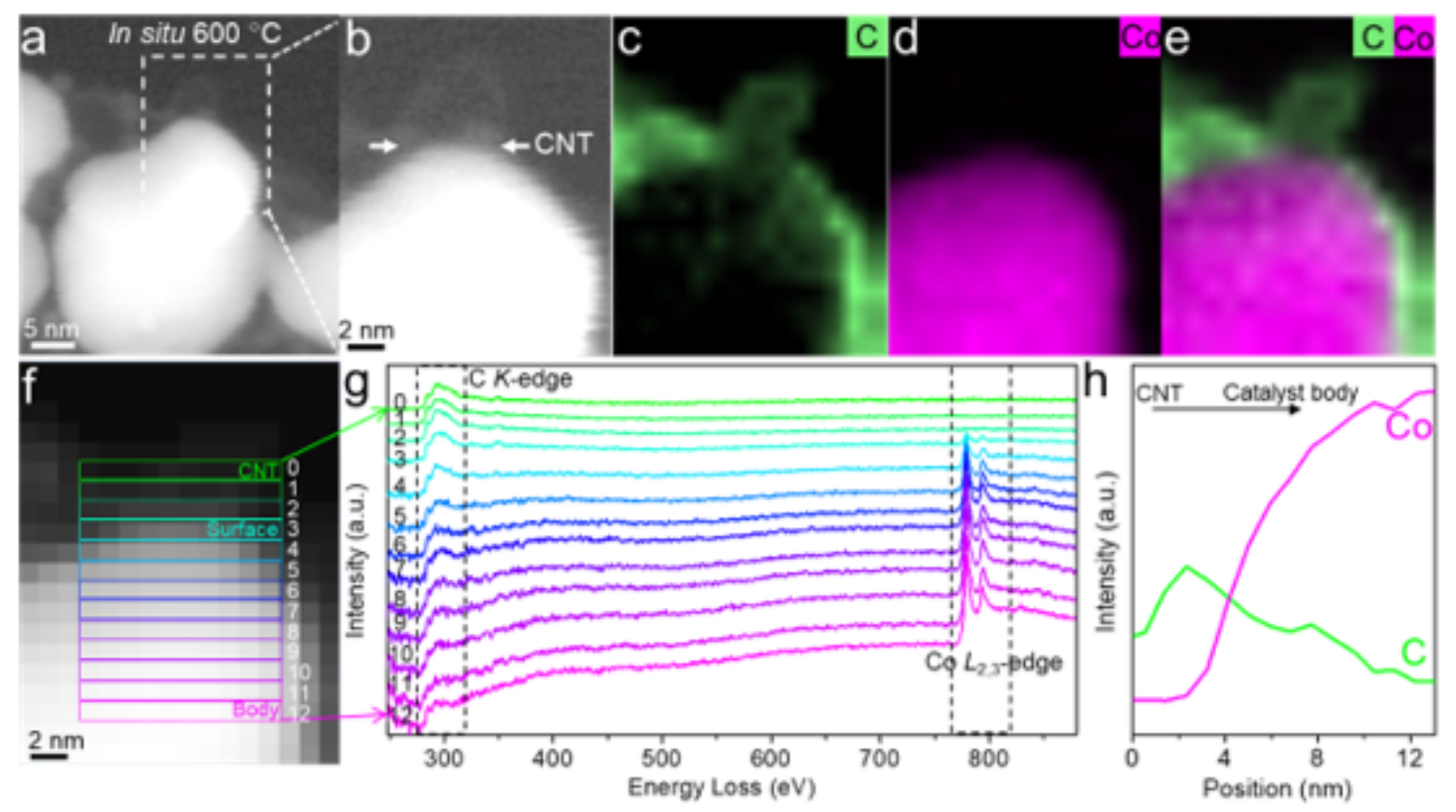

\section{Figure 5}

In-situ STEM-EELS characterization of catalyst during the growth of CNT. a, b High-angle annular dark filed (HAADF)-STEM image of a semi-suspended cobalt catalyst with a CNT grown on it (a) and the enlarged image (b). c-e Corresponding EELS-STEM elemental mapping of C K-edge (c) and Co L2,3-edge (d) on catalyst-CNT, and their combinatial image (e). Step size: $1 \mathrm{~nm}$. f, g EELS spectrum image (f), line scan EELS spectra $(\mathrm{g})$ intergrated from 0-12 layers in panel (f) across the CNT and catalyst. 0-12 marked in $(\mathrm{f})$ indicate the scan direction. Each scan area is $1 \mathrm{~nm} \times 10 \mathrm{~nm}$. Background of all spectra were subtracted using the 1 st Order Log-Polynomial model with the same rule. $\mathrm{h}$ Intensity of $\mathrm{C}$ and Co EELS signals across the CNT, catalyst surface, and body derived from (e). 

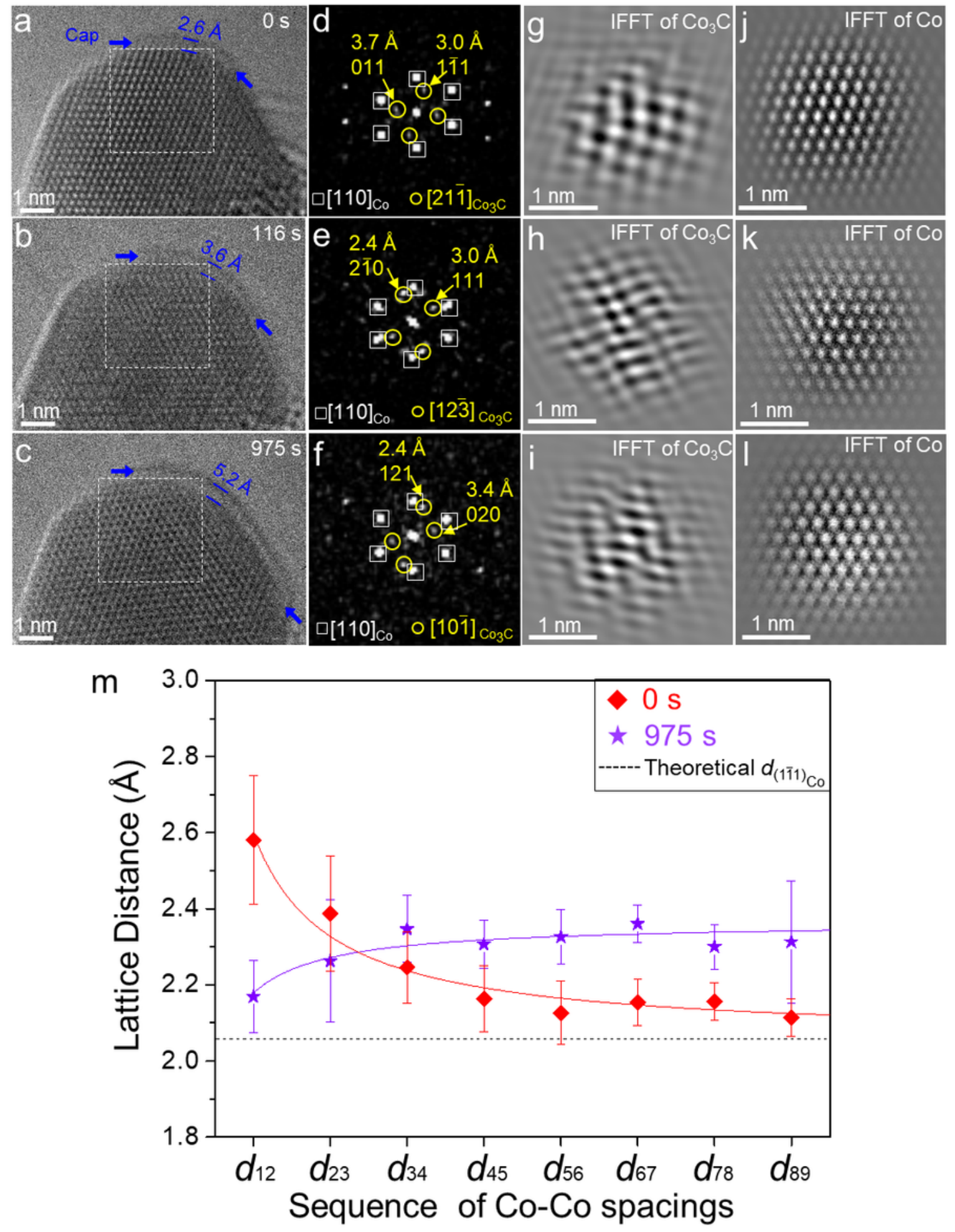

Figure 6

Structural evolution of Co catalyst during the nucleation of carbon cap. a- I Time-sequenced ETEM images of a Co nanoparticle during the growth of carbon cap under $\mathrm{CH} 4 / \mathrm{H} 2(\mathrm{v} / \mathrm{v}=7 / 3,49 \mathrm{~Pa})$ at $700{ }^{\circ} \mathrm{C}$ $(a-c)$, FFT $(d-f)$ and IFFT $(g-i)$ of the particle region marked with framework in $(a-c)$. The contrast of IFFT images $(\mathrm{i}, \mathrm{I})$ at $975 \mathrm{~s}$ were inverted due to the defocusing condition. $\mathrm{m}$ Co $(11)$ lattice spacings from surface to core region at different exposuring times. 


\section{Supplementary Files}

This is a list of supplementary files associated with this preprint. Click to download.

- SupplementaryInformation.pdf 